\title{
Fotosensibilización hepatógena en bovinos provocada por fasciolasis crónica
}

\section{Bovine hepatogenic photosensitization caused by chronic fasciolasis}

\section{P. Parodi ${ }^{1,2^{*}}$ 0000-0002-9878-2930 \\ C. Matto ${ }^{2}$ 0000-0002-7902-8676 \\ V. Rodríguez ${ }^{2}$ 0000-0002-0166-551X}
M. Schanzembach ${ }^{2}$ 0000-0002-2407-1175
E. Gianneechini ${ }^{2}$ 0000-0003-4908-9678
R. Rivero ${ }^{2}$ 0000-0003-0364-9908

${ }^{1}$ Instituto Nacional de Investigación Agropecuaria, Plataforma de Salud Animal, Tacuarembó, Ruta 5 Km 386, Tacuarembó, 45000, Uruguay. Autor para correspondencia: pabloparodi7@gmail.com ${ }^{2}$ Laboratorio Regional Noroeste, División de Laboratorios Veterinario "Miguel C. Rubino”, Ruta 3 Km 369, Paysandú, 60000, Uruguay.

Veterinaria (Montevideo) Volumen 56 № 214 (2020 Jul - Dic) e20205621403

\section{Resumen}

La fotosensibilización hepatógena en bovinos puede ser provocada por diversas causas, entre ellas, como consecuencia del daño que provoca Fasciola hepatica a nivel hepatocelular. La fotodermatitis de origen hepatógeno es uno de los signos clínicos que se puede observar en cuadros de fasciolasis crónica, debido en su capacidad de producir fibrosis hepática y colangitis con estasis biliar. El objetivo de este trabajo fue describir dos brotes de fotosensibilización hepatógena en bovinos en los departamentos de Tacuarembó y Soriano (Uruguay). La enfermedad afectó a un lote de vacas Hereford y cruza, y a un lote de vaquillonas cruzas. En ambos brotes los animales pastoreaban en campos forestales húmedos. Los principales signos clínicos observados fueron pérdida de peso, costras y peladuras en morro, fotofobia, sialorrea y corrimientos oculares. Se realizaron necropsias visualizando como hallazgos macroscópicos relevantes dermatitis necrótica bilateral de los párpados, úlceras en cara ventral de lengua. En hígado se observó engrosamiento de la cápsula de Glisson, fibrosis difusa y colangitis crónica con calcificación de los canalículos biliares. Al examen histopatológico se destacó como principal lesión, fibrosis hepática y obstrucción canalicular con presencia de Fasciola hepatica. En base a los signos clínicos, hallazgos macro y microscópicos, la fotosensibilización observada en los animales de ambos brotes estuvo directamente relacionada con las alteraciones hepáticas crónicas causadas por F. hepatica.
Recibido: $23 / 07 / 2020$

Aceptado: 09/09/2020 (cc) EY-NO

\section{Abstract}

Bovine hepatogenic photosensitization can be caused by several causes, such as hepatocellular damage due to by Fasciola hepatica. Hepatogenic photodermatitis is one of the clinical signs that can be observed in chronic fasciolasis, caused by hepatic fibrosis and cholangitis with bile stasis. The objective of this work was to describe two outbreaks of hepathogenic photosensitization in cattle in the counties of Tacuarembó and Soriano (Uruguay). The disease affected a herd of Hereford and crossbreed cows and a herd of crossbreed heifers. In both outbreaks the animals grazed in damp forest fields. The main clinical signs observed were weight loss, scabs and peeling on the snout, photophobia, hypersalivation and ocular bleeding. In all necropsies performed, the relevant macroscopic findings were bilateral necrotic dermatitis of the eyelids, ulcers on the ventral surface of the tongue. In the liver, there was thickening of the Glisson's capsule, diffuse fibrosis, and chronic cholangitis with calcification of the bile canaliculi. Histopathologically the main lesion was liver fibrosis and canalicular obstruction with the presence of $F$. hepatica. Based on the clinical signs, macro and microscopic findings, the photosensitization observed in the animals of both outbreaks was directly related to the chronic liver alterations caused by F. hepatica.

Keyword: bovine photodermatitis, phylloerythrin, chronic distomatosis.

Palabras claves: fotodermatitis bovina, filoeritrina, distomatosis crónica. 


\section{Introducción}

La fotosensibilización en bovinos es una patología que ocurre cuando pigmentos fluorescentes o fotodinámicos se depositan en la piel, éstos absorben rayos UV causando muerte celular local y edema tisular (Casteel et al., 1991). Los principales signos clínicos observados son: intensa irritación de la piel, prurito, laceraciones y edemas en zonas despigmentadas, cierre de párpados, lagrimeo, disfagia, sialorrea y fotofobia (Rowe, 1989). Esta patología tiene tres posibles orígenes. En primer lugar, fotosensibilización primaria, causada por la ingesta de agentes fotodinámicos exógenos, como por ejemplo, intoxicaciones por Ammi majus y A. viznaga (Perusia y Rodriguez, 2017). Fotosensibilización secundaria causada por la acumulación de pigmentos endógenos, como son los casos de porfíria congénita (Rowe, 1989). Por último, fotosensibilización hepatógena, provocado por el acúmulo del pigmento filoeritrina en la circulación periférica. Éste es un producto de la digestión de la clorofila que normalmente es excretada en la bilis. Pero cuando hay lesiones hepáticas graves con pérdida de la funcionalidad del órgano $\mathrm{u}$ obstrucciones canaliculares con estasis biliar, la excreción de este pigmento se ve afectada, pasando a la circulación periférica y alcanzando la piel (Glen et al., 1964).

La funcionalidad hepática puede verse alterada debido a lesiones en hígado, que comúnmente pueden ser producidas por plantas hepatotóxicas, hongos, químicos o parásitos (Smith et al., 1997). Dentro de los parásitos, la fasciolasis, causada por Fasciola hepatica, provoca graves lesiones en el hígado de los rumiantes (Adrien et al., 2013). Estudios de seroprevalencia de F. hepatica realizados en Uruguay reportan alta prevalencia tanto a nivel predial como individual (Sanchis et al., 2015), siendo una enfermedad relevante para la producción ganadera.

La fasciolasis en bovinos puede presentarse de diferentes formas (subaguda, aguda y crónica) dependiendo de la cantidad de metacercarias ingeridas y del grado de resistencia de los bovinos (Adrien et al., 2013). Cuando los animales ingieren metacercarias de $F$. hepatica, éstas se enquistan en el duodeno transformándose en formas inmaduras que penetran la pared intestinal, cruzan la cavidad peritoneal y migran hasta el hígado atravesando la cápsula de Glisson (Aksoy et al., 2005). En el parénquima hepático, migran hasta los ductos biliares, causando lesiones traumáticas, produciendo trayectos hemorrágicos y necrosis coagulativa (Dow et al., 1968).

La injuria causada por la migración de larvas, activan las células estelares del hígado, sintetizando colágeno de tipo I, III, IV, además de proteoglicanos, laminina, fibronectina y ácido hialurónico, provocando fibrosis hepática (Marcos et al., 2007). Los parásitos maduros se alojan en los canalículos biliares, causando colangitis crónica, mineralización y engrosamiento de sus paredes, que en su interior contiene una sustancia negra amarronada, constituida por bilis anormal y detritos celulares (Dow et al., 1968; Marcos et al., 2007), provocando una estenosis ductal con estasis biliar. El objetivo de este trabajo fue describir dos brotes de fotosensibilización hepatógena en bovinos, relacionado a lesiones crónicas de fibrosis causadas por $F$. hepatica.

\section{Materiales y métodos}

E1 Laboratorio Regional Noroeste de la División de Laboratorios Veterinarios (DILAVE, Paysandú, Uruguay) "Miguel C. Rubino" fue consultado en dos oportunidades por brotes de fotosensibilización de origen desconocido con muertes de bovinos.

\section{Descripción de los brotes}

Brote A: Afectó a un lote de 153 vacas entre 3 y 4 años (Hereford y cruzas) localizadas en el departamento de Soriano (Uru-

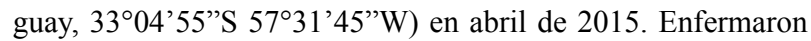
14 vacas y murieron $14(9 \%)$ en un periodo de 60 días. Los animales se encontraban pastoreando en un establecimiento ganadero forestal, cercano a la represa del Palmar, zona húmeda, con dos lagunas de buen caudal de agua. Dicho predio tenía antecedentes de $F$. hepatica, en el cual se había dosificado con Nitroxinil al $34 \%(6,8 \mathrm{mg} / \mathrm{kg})$ dos meses antes del brote.

Brote B: Afectó a un lote de 150 vaquillonas (cruza) de 2 años localizadas en el departamento de Tacuarembó (Uruguay, $32^{\circ} 12^{\prime} 14^{\prime \prime}$ S 545' $32^{\prime \prime} \mathrm{W}$ ) en febrero de 2018. Enfermaron 30 $(33,3 \%)$ y murieron $3(2 \%)$, en un periodo de evolución 30 días. Se encontraban pastoreando un campo forestal, con poca disponibilidad de forraje y un tajamar con buena disponibilidad de agua. Las vaquillonas fueron dosificadas con Ivermectina $3,15 \%(0,63 \mathrm{mg} / \mathrm{kg}$ un mes previo al brote y con Nitroxinil al $34 \%(6,8 \mathrm{mg} / \mathrm{kg})$ dos meses previos al brote.

En ambos predios se recorrió un 70\% de la superficie, para verificar la existencia de plantas tóxicas, no encontrando especies de probada toxicidad hepática.

\section{Examen clínico y necropsia}

En la visita a ambos predios se describieron los principales signos clínicos de los animales enfermos y se realizaron 4 necropsias (dos animales por brote). Se registraron las alteraciones macroscópicas relevantes observadas en las necropsias, como cambios en la coloración de los órganos, tamaño, forma y consistencia. Se tomaron muestras de órganos (hígado, bazo, riñón, intestino delgado y grueso, corazón, pulmón, ganglios mesentéricos, lengua, esófago, sistema nervioso central) para su estudio histopatológico.

\section{Histopatología}

Los órganos obtenidos en las necropsias fueron fijados en formol bufferado al 10\%, luego embebidos en parafina, cortados a $5 \mu \mathrm{m}$ de espesor y coloreados con hematoxilina y eosina (HE) (AFIP, 1995). 


\section{Pruebas bioquímicas}

Se tomaron muestras de sangre obtenida de la vena coccígea, de animales con signos de fotodermatitis (4 animales brote A y 9 animales brote B). Se realizaron mediciones en suero de Proteínas totales (PT), Albúmina (Alb), Aspartato Aminotransferasa (AST), Gama Glumatil Transpeptidasa (GGT) y Fosfatasa Alcalina (FAS). Para las determinaciones de proteínas y enzimas se utilizó un equipo de bioquímica clínica Dimension ${ }^{\circledR} \mathrm{R} l \mathrm{x}$ max (Siemens), técnica colorimétrica con espectrofotometría.

\section{Resultados}

\section{Signos clínicos}

En la visita a ambos predios se constataron:

Brote A. Seis animales de los 14 enfermos presentaron movimiento de cabeza, dermatitis en morro, edema palpebral, lesiones ulcerativas en cara ventral de lengua. Otros animales enfermos mostraron ictericia, diarrea oscura, desmejoramiento de su estado corporal, pelo hirsuto.

Brote B. Se observaron 10 animales con alopecias y costras en morro, sialorrea, corrimiento ocular, dermatitis en párpados y en otras áreas blancas, miasis en zona ocular (Fig. 1). Algunos también presentaban edema submandibular y diarrea.
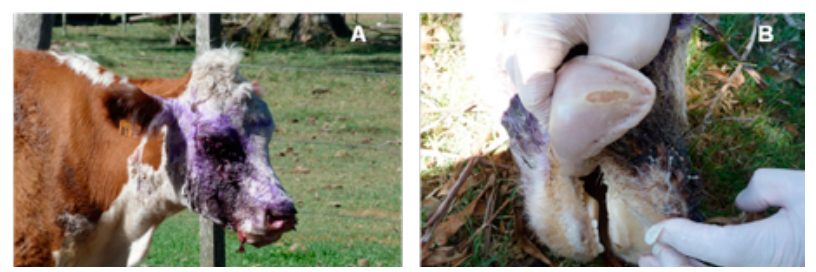

Figura 1: Vaquillonas pertenecientes al brote B. Foto A, corrimiento ocular, con dermatitis necrótica de los párpados. Desprendimiento de piel en zona de morro.

Foto $\mathrm{B}$, úlcera en región ventral de lengua.
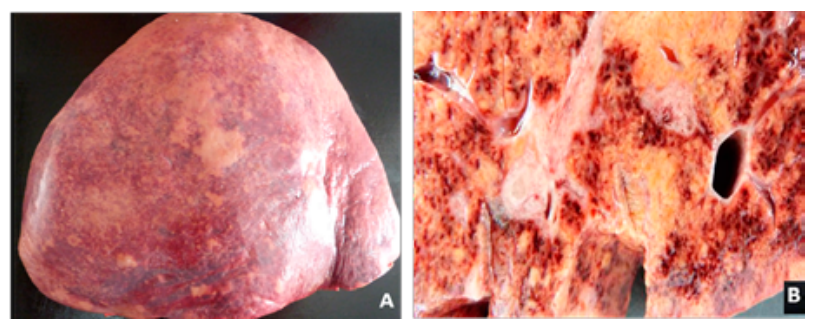

Figura 2: Vaca perteneciente al brote A. Hígado: marcada alteración en la coloración del órgano. Foto A, se presentaban áreas oscuras intercaladas por áreas claras en la superficie diafragmática. Foto B, en la superficie de corte se observan áreas multifocales de coloración amarillenta, correspondientes a zonas de necrosis coagulativa, fibrosis y engrosamiento de los canalículos.

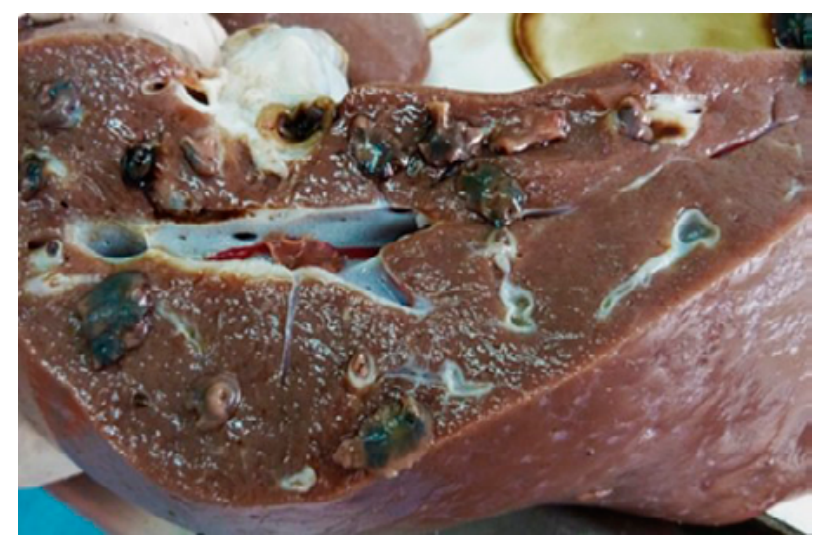

Figura 3: Brote B. Vaquillona. Hígado: áreas oscuras, hemorrágicas intercaladas con áreas claras, sustitución del parénquima hepático por tejido fibroso y engrosamiento de los canalículos. Se visualizan $F$. hepatica adultas (flechas).

\section{Hallazgos de necropsia}

En las 4 necropsias realizadas los principales hallazgos macroscópicos fueron: moderada ictericia, dermatitis necrótica bilateral de los párpados, con corrimiento supurativo y úlceras en cara ventral de lengua (Fig. 1). En hígado se observó cambio de coloración (amarillo ocre), engrosamiento de la cápsula de Glisson, fibrosis difusa, severa, con sustitución del parénquima hepático y aumento de la consistencia al corte. Colangitis crónica con calcificación de los canalículos y presencia de F. hepatica (Fig. 2 y 3 ).

\section{Hallazgos histológicos}

Las principales lesiones histopatológicas se encontraron a nivel de hígado, observándose fibrosis de la cápsula de Glisson, áreas multifocales extensas de necrosis coagulativa e infiltrado inflamatorio a predominio linfoeosinofílico. Sustitución del parénquima hepático por tejido fibroso, colangitis crónica con proliferación de los ductos biliares y calcificación canalicular asociado a fibrosis pericanalicular e hiperplasia glandular con presencia de F. hepatica intracanalicular (Fig. 4).

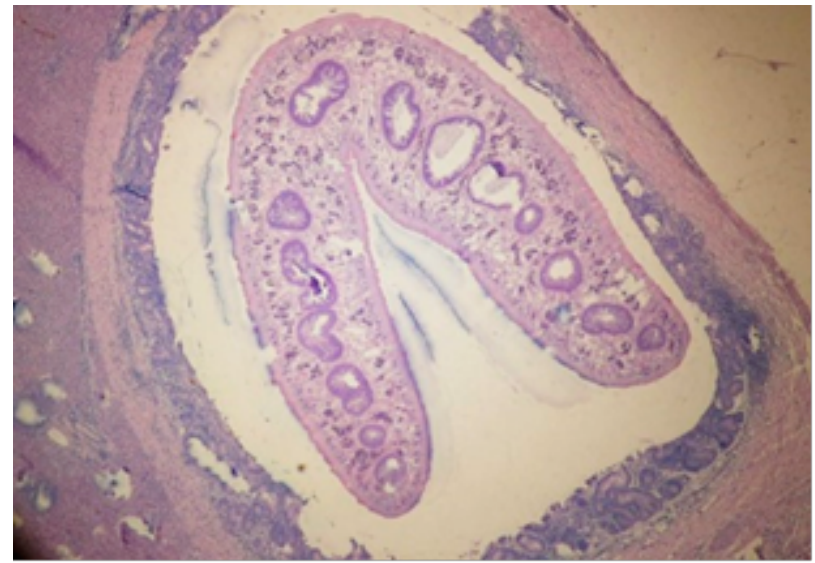

Figura 4: Brote B. Vaquillona. Hígado: colangitis crónica, fibrosis pericanalicular, hiperplasia glandular (flecha) y presencia de forma adulta de Fasciola hepatica intracanalicular $(\mathrm{H} \& \mathrm{E}, 100 \times)$ 
Otras alteraciones histológicas registradas fueron: en porción ventral de lengua úlceras focalmente extensas del epitelio con tejido de granulación. En zonas de piel despigmentadas expuestas a la luz solar, se observó en epidermis áreas multifocales extensas de necrosis coagulativa, reacción inflamatoria supurativa, con formación de costras. En dermis, edema difuso con moderada infiltración inflamatoria supurativa. Se visualizó a nivel renal degeneración tubular moderada difusa, con infiltrado linfoplasmocítico intersticial multifocal discreta. En tracto digestivo, se constató gastroenterocolitis difusa con edema de la submucosa a nivel de abomaso e intestino delgado. En los demás órganos no se observaron lesiones de significación.

\section{Funcional hepático}

Brote A (Tabla 1), en 3 animales (3/4) los resultados indicaron altos niveles de proteína total, mientras que la albúmina superaba los parámetros de referencia en 2 vacas (2/4). Por otro lado, en la totalidad de los animales (4/4) los niveles AST, GGT y FAS se encontraban por encima de los valores de referencia.

En el brote B, se observó en todos los animales muestreados (9/9) niveles de proteínas totales y GGT normales, mientras que los valores de AST estaban aumentados. La albúmina en 4 animales (4/9) se encontró por encima de los niveles normales. Por otro lado, los valores de FAS estaban alterados en 4 de ellos (4/9) (Tabla1).

Tabla 1: Resultados de pruebas bioquímicas sanguíneas (A) y (B): Identificación del brote. PT: Proteínas g/l (referencia 62-90), Alb: Albumina U/L (referencia 30-36), AST: Aspartato aminotransferasa U/L (referencia 40-90), GGT: Gamma Glutamil Transpeptidasa U/L (referencia 2-20), Fas: Fosfatasa Alcalina $\mathrm{U} / \mathrm{L}($ referencia $<225)$.

\begin{tabular}{lllllll}
\hline & Animal & PT & Alb & AST & GGT & FAS \\
\hline & 1 & 120 & 42 & 250 & 76 & 250 \\
Brote A & 2 & 103 & 38 & 136 & 166 & 230 \\
& 3 & 89 & 37 & 176 & 131 & 370 \\
& 4 & 101 & 35 & 186 & 206 & 227 \\
& & & & & & \\
Brote B & 1 & 74 & 34 & 115 & 9 & 352 \\
& 2 & 70 & 39 & 135 & 9 & 277 \\
& 3 & 75 & 36 & 126 & 16 & 136 \\
& 5 & 71 & 40 & 103 & 10 & 293 \\
& 6 & 72 & 34 & 92 & 10 & 77 \\
& 7 & 76 & 36 & 156 & 7 & 140 \\
& 8 & 59 & 32 & 101 & 9 & 85 \\
& 9 & 75 & 43 & 115 & 17 & 374 \\
\hline
\end{tabular}

\section{Discusión}

En Uruguay existe un incremento muy importante en los últimos años de focos de fasciolasis, en relación con condiciones climáticas crecientemente cálidas y de mayor precipitación acumulada anual (Matto et al., 2016). Esto ha hecho posible la observación de casos frecuentes de fotosensibilización hepatógena a causa de las lesiones hepáticas provocadas por F. hepatica. Los signos clínicos que presentaron los animales de este estudio, correspondieron a un cuadro de fotosensibilización, tales como: desprendimiento de la piel del morro y párpados, edema alrededor de los ojos, lagrimeo bilateral, movimiento de cabeza, fotofobia (Collett y Matthews, 2014). Estos signos de fotosensibilización se podrían atribuir al acúmulo de filoeritrina (producto de la metabolización de la clorofila), causada por la estasis biliar provocada por la colangitis y obstrucción biliar que producen las lesiones crónicas de F. hepatica (Perusia y Rodriguez, 2017; Cardona et al., 2015; Olaechea et al., 2011; Smith et al., 1997). Las principales lesiones que se destacaron en los animales fueron a nivel hepático, donde se observó macro y microscópicamente lesiones de un cuadro de fasciolasis. Según lo reportado por Dow et al. (1968), en base a las lesiones histopatológicas, ambos brotes de fotosensibilización correspondieron a un cuadro de fasciolasis crónica. Teniendo en cuenta la fibrosis hepática, visualización de morfologías parasitarias adultas de $F$. hepatica dentro de los canalículos biliares, colangitis, obstrucción biliar, fibrosis periportal difusa, degeneración de hepatocitos y proliferación canalicular.

Estas lesiones se vieron reflejadas en las pruebas bioquímicas sanguíneas, observando la actividad catalítica de las enzimas hepáticas, en particular la GGT y la AST. Un aumento considerable de las concentraciones séricas de estas enzimas es indicador de alteración hepática, como lo registrado en ambos brotes (Ocal et al., 2013). En especial la GGT (ubicada a nivel de los canalículos hepáticos) asociada a un aumento de la Fosfatasa Alcalina indican una colangitis y colestasis biliar (Gracindo et al., 2014). Dentro de los agentes que provocan fotosensibilización en bovinos, se debe considerar otros causales de fibrosis hepáticas como intoxicaciones por Senecio spp, Echium plantagineum, Erechtites hieracifolia (Cardona et al., 2015; Galitzer y Oehme, 1978). Estas intoxicaciones se caracterizan por generar alteraciones histológicas diferentes a fasciolasis, destacando megalocitosis, fibrosis y proliferación canalicular difusa (Preliasco y Rivero, 2011). Las micotoxinas como la Aflatoxina B1, también pueden producir fibrosis hepática (Cullen y Stalker, 2016), aunque se encuentran frecuentemente relacionadas con suministro de alimentos concentrados o granos, por lo que se descartó, ya que los animales pastoreaban sólo campo natural.

Como otra causa de fotosensibilización hepatógena, se encuentra la intoxicación por Phytomices chartarum (Cardona et al., 2015). El diferencial se realiza en base a condiciones epidemiológicas, ya que los animales pastoreaban en campo natural con bajo nivel de forraje, situación contraria a las 
condiciones adecuadas para el desarrollo del hongo, las cuales necesitan pasturas exuberantes, praderas, rastrojos, con abundante materia muerta. A su vez esta intoxicación cursa con un cuadro histológico distinto, predominando la colangitis y fibroplasia periportal que permite diferenciar con lo ocasionado por F. hepática (Tokarnia et al., 2012; Smith y Embling, 1991).

También la literatura menciona intoxicaciones por plantas que contienen saponinas esteroidales litogénicas como Brachiaria decumbens o Panicum spp.. Estas causan una insuficiencia hepática, induciendo migración de macrófagos espumosos y estimulando la formación de cristales en los hepatocitos y ductos biliares que obstruyen los canalículos biliares con el consecuente acúmulo de filoeritrina. Panicum spp está presente en Uruguay, mientras que Brachiaria decumbens es una gramínea forrajera altamente utilizada en Brasil, muy adaptada a climas tropicales y sueles de baja fertilidad (Cardona et al., 2015). Estas intoxicaciones se descartaron ya que a las lesiones histopatológicas son diferentes, además de que no se detectó la presencia de estas gramíneas en los predios. Otras plantas que producen cuadros de fotosensibilización hepatógena reportadas en Uruguay, son Lantana camara y Myoporum laetum. Estas presentan diferentes cuadros epidemiológicos y lesiones histopatológicas características, sin un patrón de fibrosis hepática difusa (Tokarnia et al., 2012, Rivero et al., 2011).

\section{Conclusión}

En base a los signos clínicos, lesiones macro y microscópicas permitió realizar un diagnóstico de fotosensibilización hepatógena en ambos brotes. La fotosensibilización observada en los animales estuvo directamente relacionada a la disfunción hepática provocada por lesiones crónicas de Fasciola hepatica. Frente a cuadros de fotosensibilización en bovinos es necesario integrar entre las posibles causas a aquellos agentes que producen lesiones hepáticas severas como las de F. hepatica. La necropsia completa de los animales afectados continúa siendo la principal herramienta diagnóstica para el veterinario.

\section{Agradecimientos}

Se expresa especial agradecimiento al Laboratorio Regional Noroeste de la División de Laboratorios Veterinarios (DILAVE, Paysandú, Uruguay) "Miguel C. Rubino" por la realización y financiamiento de este trabajo/estudio.

LOS AUTORES DECLARAN NO TENER CONFLICTOS DE INTERES

\section{Referencias}

Adrien, M.L., Schild, A.L., Marcolongo-Pereira, C., Fiss, L., Ruas, J.L., Grecco, F.B. y Raffi, M.B. (2013). Acute fasciolasis in cattle in southern Brazil. Pesquisa Veterinaria Brasileira, 33(6), 705-709. doi: 10.1590/S0100736X2013000600003

AFIP (1995). Métodos Histotecnológicos. Washington: Registro de Patología de los Estados Unidos de América.

Aksoy, D.Y., Kerimoglu, U., Oto, A., Erguven, S., Arslan, S, Unal, S... Bayraktar, Y. (2005). Infection with Fasciola hepatica. Clinical Microbiological and Infectious, 11(11), 859861. doi: 10.1111/j.1469-0691.2005.01254.x

Cardona, J., Vargas, M. y Paredes E. (2015). Estudio clínico e histopatológico de la dermatitis fototóxica en terneros Cebu en pastoreo de Bachiaria decumbens. Revista de Medicina Veterinaria y Zootecnia, Cordoba, 21(2), 5366-5380.

Casteel, S.W., Weaver, A.D., Mills, L.L., Pace, L.W., Rottinghaus, G.E. y Smith, K.M. (1991). Photosensitization outbreak in Shorthorn calves in Missouri. Journal of Veterinary Diagnostic Investigation, 3(2), 180-182. doi: 10.1177/104063879100300218

Collett, M. y Matthews, Z.M. (2014). Photosensitivity in Cattle Grazing Brassica Crops. International Journal of Poisonous Plant Research, 3(1), 6-21. Recuperado de https://www.researchgate.net/publication/289996856

Cullen, J. y Stalker, M. (2016). Liver and biliary system. En: Jubb, Kennedy y Palmer's. Pathology of domestic animals ( 6 a ed., Vol. 2, pp. 258-351). Ontario: Grant Maxie.

Dow, C., Ross, J.G. y Todd, J.R. (1968). The histopathology of Fasciola hepatica infections in sheep. Parasitology, 58(1), 129-135. doi: 10.1017/S0031182000073480

Galitzer, S.J. y Oehme, F.W. (1978). Photosensitization: literature review. Veterinary Research Communications, 2(1), 217-230. doi: 10.1007/BF02291451

Glen, B.L., Monlux, A.W. y Panciera, R.J. (1964). Hepatogenous photosensitivity disease of cattle. Veterinary Pathology, 1, 469-484. doi: 10.1177/030098586400100601

Gracindo, C., Louvandini, H., Riet-Correa, F., Barbosa-Ferreira, M. y Botelho de Castro, M. (2014). Performance of sheep grazing in pastures of Brachiaria decumbens, Brachiaria brizantha, Panicum maximum, and Andropogon gayanus with different protodioscina concentrations. Tropical Animal Health and Production, 46, 733-737. doi: 10.1007/s11250- 
014-0556-y

Marcos, L.A., Yi, P., Machicado, A., Andrade, R., Samalvides, F., Sanchez, F. y Terashima, A. (2007). Hepatic fibrosis and Fasciola hepatica infection in cattle. Journal of Helminthology, 81, 381-386. doi: 10.1017/s0022149x07850231

Matto, C., Adrien, M.L., Ceriani, S., Giannechini, E., Buroni, F. y Rivero, R. (2016). Estudio retrospective de la fasciolasis bovina durante el periodo de 1998 a 2015 en el litoral noroeste. En: XLIV Jornadas Uruguayas Buiatría (pp 229-231). Paysandú: Centro Médico Veterinario Paysandú.

Olaechea, F., Lovera, V., Larroza, M., Raffo, F. y Cabrera, R. (2011). Resistance of Fasciola hepatica against triclabendazole in cattle in Patagonia (Argentina). Veterinary Parasitology, 178, 364-3. doi: 10.1016/j.vetpar.2010.12.047

Ocal, N., Haydardedeoğlu, A., Cinar, M., Kul, O. y Türk, M. (2013). Hepatogenous photosensitization in Akkaraman lambs: special emphasis to oxidative stress and thrombocytopenia. Ankara Üniversitesi Veteriner Fakültesi Dergisi, 60, 117-122.

Perusia, O.R. y Rodriguez, R. (2017). Fotosensibilización. Plantas toxicas y micotoxinas. [Archivo electrónico]. Recuperado de www.produccion-animal.com.ar

Preliasco, M. y Rivero, R. (2011). Poisoning of cattle by Senecio spp. in Uruguay. En F. Riet-Correa, J. Pfister, T. Wierenga y A.L. Schild, Poisoning by plants, mycotoxins and related toxins (pp 198-207). Wallingford: CAB International.

Rivero, R., Gianneechini, E., Matto, C. y Gil, J. (2011). Intoxicación por Lantana camara en bovinos y ovinos en Uruguay. Veterinaria Montevideo, 47(181), 29-34.

Rowe, L.D. (1989). Photosensitization problem in livestock. Veterinary Clinics North America: Food animal practice, 5(2), 301- 323. doi: https://doi.org/10.1016/S07490720(15)30978-6

Sanchis, J., Hillyer, G.V., Madeira de Carvalho, L.M., Macchi, M.I., Gomes, C., Maldini, G... Arias, M.S. (2015). Riesgo de exposición a Fasciola hepatica en ganado vacuno en extensivo de Uruguay y Portugal determinado mediante ELISA y un antígeno recombinante. Archivos de Medicina Veterinaria, 47, 201-208. doi: 10.4067/S0301-732X2015000200011

Smith, B.L., Asher, G.W., Thomposon, K.G. y Hoggard, G.K. (1997). Hepatogenous phosensitisation in fallw deer (Dama dama) in New Zeland. New Zealand Veterinary Journal, 45(3), 88-92. doi: 10.1080/00480169.1997.36001 gy. New Zealand Veterinary Journal, 39(1), 18-22. doi: 10.1080/00480169.1991.35650

Tokarnia, C.H., de Farias, M., Diomedes, J., Peixoto, P.V. y Döbereiner, J. (2012). Plantas/ Micotoxinas fotosesensibilizantes. En C.H. Tokarnia, M. de Farias, J. Diomedes, P.V. Peixoto y J. Döbereiner. Plantas tóxicas do Brasil para animais de produção (2a ed., pp. 305-341). Rio de Janeiro: Helianthus,

\section{Nota de contribución:}

P. Parodi (36\%). Diseño del estudio. Análisis e interpretación de los datos. Redacción del manuscrito. Revisión crítica del manuscrito. Dio la aprobación final

C. Matto (20\%). Análisis e interpretación de los datos. Revisión crítica del manuscrito. Dio la aprobación final

V. Rodríguez (8\%). Revisión crítica del manuscrito.

M. Schanzembach (8\%). Revisión crítica del manuscrito.

E. Gianneechini (8\%). Revisión crítica del manuscrito.

R. Rivero (20\%). Diseño del estudio. Análisis e interpretación de los datos. Revisión crítica del manuscrito. Dio la aprobación final.

El editor Cecilia Cajarville aprobó ese artículo.

Smith, B.L. y Embling, P.P. (1991). Facial eczema in goats:

The toxicity of sporidesmin in goats and its patholo- 